\title{
ON MARKOV STABILITY
}

\author{
CORRADO RISITO 1
}

\begin{abstract}
The concept of $T$-stability for vector-valued functions is introduced-a generalization of strong stability in the sense of Markov. Moreover, for solutions of $T$-periodic systems of differential equations, $T$-stability is compared with Liapunov stability and it is shown that boundedness and $T$-stability imply asymptotic almost periodicity.
\end{abstract}

1. Introduction. Markov defined strong stability in the following way: the function $x: \mathbf{R} \rightarrow \mathbf{R}^{n}$ is called strongly stable if, for every $\varepsilon>0$, there is a $\delta>0$ such that if $\alpha$ and $\beta$ are any two real numbers with $\|x(\alpha)-x(\beta)\|<$ $\delta$, then $\|x(t+\alpha)-x(t+\beta)\|<\varepsilon$ for all $t \in \mathbf{R}$ [1] (by the way, he also defined strong stability in the past and strong stability in the future). The importance of this concept results from the Markov theorem: if the function $x(t)$ is continuous, bounded and strongly stable, then $x(t)$ is almost periodic [1] (see also [2, p. 181]).

In the present paper we define a weaker type of stability, requiring that Markov condition holds only for $\alpha$ and $\beta$ integral multiples of some real number $T>0$ (independent of $\varepsilon$ ), and distinguish between $T$-stability in the past and in the future (see Definition 1 of \$2) and T-stability in the future only (shortly $T$-stability) (see Definition 2 of \$2). Moreover, we establish the following theorems (Theorems 1 and 2): if the function $x(t)$ is uniformly continuous and bounded, then $T$-stability in the past and in the future ( $T$-stability) implies that $x(t)$ is almost periodic (asymptotically almost periodic). Theorem 1 is not a generalization of the Markov theorem, because we assume the uniform continuity of $x(t)$. As a matter of fact Theorems 1 and 2 become interesting especially in the theory of periodic systems of differential equations, because boundedness of a solution $x(t)$ implies the uniform continuity of $x(t)$. Our main result is (Theorem 3): if a solution $x(t)$ of a $T$-periodic system is bounded in the future and $T$-stable, then $x(t)$ is asymptotically almost periodic. At last we show that $T$-stability of a solution $x(t)$ of a $T$-periodic system of differential equations is nothing else than conditional stability of $x(t)$ with respect to the solutions $x(t+m T), m=$ $1,2, \ldots$ Therefore $T$-stability is weaker than Liapunov stability, and by Theorem 3 we obtain the Corollary of $\$ 3$ which shows that the condition of uniform Liapunov stability, usually adopted in the literature to assure

Received by the editors December 10, 1977.

AMS (MOS) subject classifications (1970). Primary 34C25; Secondary 34D20.

${ }^{1}$ The research of this paper was supported by the "Consiglio Nazionale delle Ricerche" of Italy (C.N.R.-G.N.F.M.). 
asymptotic almost periodicity, is unnecessarily strong.

2. Definition of $T$-stability. Let $x(t)$ be a vector-valued function of the real variable $t$, defined on the real line $\mathbf{R}$ with values in $\mathbf{R}^{n}$ (the Euclidean space of dimension $n$ ) and let $\|\cdot\|$ designate any norm on $\mathbf{R}^{n}$. We give the following

Definition 1. The function $x(t)$ is called $T$-stable in the past and in the future if there exists a $T>0$ such that

$$
\begin{gathered}
(\forall \varepsilon>0)(\exists \delta>0)(\forall n, m \in \mathbf{Z}:\|x(n T)-x(m T)\|<\delta)(\forall t \in \mathbf{R}) \\
\|x(t+n T)-x(t+m T)\|<\varepsilon,
\end{gathered}
$$

where $\mathbf{Z}$ is the set of relative integers.

The following theorem holds

THEOREM 1. If the function $x: \mathbf{R} \rightarrow \mathbf{R}^{n}$ satisfies the following conditions

(i) $x(t)$ is uniformly continuous and bounded on $\mathbf{R}$,

(ii) $x(t)$ is $T$-stable in the past and in the future (according to Definition 1), then $x(t)$ is almost periodic.

Let $\left\{\alpha_{n}\right\}$ be a sequence of real numbers. Then for every $n(n=1,2, \ldots)$ there is $k_{n} \in \mathbf{Z}$ such that $k_{n} T \leqslant \alpha_{n}<\left(k_{n}+1\right) T$. Put $\beta_{n}=\alpha_{n}-k_{n} T$. Owing to the boundedness of the sequences $\left\{\beta_{n}\right\}$ and $\left\{x\left(k_{n} T\right)\right\}$, we can extract two common subsequences of $\left\{\beta_{n}\right\}$ and $\left\{k_{n}\right\}$, respectively denoted by $\left\{\beta_{n}^{\prime}\right\}$ and $\left\{k_{n}^{\prime}\right\}$, such that the sequences $\left\{\beta_{n}^{\prime}\right\}$ and $\left\{x\left(k_{n}^{\prime} T\right)\right\}$ are convergent. Let us consider the subsequence $\left\{\alpha_{n}^{\prime}\right\}$ of $\left\{\alpha_{n}\right\}$, where $\alpha_{n}^{\prime}=\beta_{n}^{\prime}+k_{n}^{\prime} T$. We prove now that the sequence of functions $\left\{x\left(t+\alpha_{n}^{\prime}\right)\right\}$ is uniformly convergent on $\mathbf{R}$, i.e. $x(t)$ is almost periodic like Bochner. In fact, for a given $\varepsilon>0$, there correspond $\delta_{1}=\delta_{1}(\varepsilon)>0$ and $\delta_{2}=\delta_{2}(\varepsilon)>0$ such that the conditions of uniform continuity and $T$-stability in the past and in the future of $x(t)$ are respectively satisfied. Furthermore there exists a positive integer $N=N(\varepsilon)$ such that for all $n, m \geqslant N$ it is: $\left|\beta_{n}^{\prime}-\beta_{m}^{\prime}\right|<\delta_{1},\left\|x\left(k_{n}^{\prime} T\right)-x\left(k_{m}^{\prime} T\right)\right\|<\delta_{2}$. Then for all $n, m \geqslant N$ and all $t \in \mathbf{R}$ we have

$$
\begin{aligned}
\left\|x\left(t+\alpha_{n}^{\prime}\right)-x\left(t+\alpha_{m}^{\prime}\right)\right\| & \leqslant\left\|x\left(t+\beta_{n}^{\prime}+k_{n}^{\prime} T\right)-x\left(t+\beta_{N}^{\prime}+k_{n}^{\prime} T\right)\right\| \\
& +\left\|x\left(t+\beta_{N}^{\prime}+k_{n}^{\prime} T\right)-x\left(t+\beta_{N}^{\prime}+k_{m}^{\prime} T\right)\right\| \\
& +\left\|x\left(t+\beta_{N}^{\prime}+k_{m}^{\prime} T\right)-x\left(t+\beta_{m}^{\prime}+k_{m}^{\prime} T\right)\right\|<3 \varepsilon,
\end{aligned}
$$

which proves the theorem.

Let $x(t)$ be a vector-valued function defined on some interval $\left[t_{0}, \infty\right)$. We introduce the concept of $T$-stability in the future, shortly denoted by $T$ stability, as follows

Definition 2. The function $x:\left[t_{0}, \infty\right) \rightarrow \mathbf{R}^{n}$ is called $T$-stable if there exists a $T>0$ such that

$$
\begin{gathered}
(\forall \varepsilon>0)(\exists \delta>0)\left(\forall n, m \in \mathrm{N}:\left\|x\left(t_{0}+n T\right)-x\left(t_{0}+m T\right)\right\|<\delta\right)\left(\forall t \geqslant t_{0}\right) \\
\|x(t+n T)-x(t+m T)\|<\varepsilon,
\end{gathered}
$$

where $\mathbf{N}$ is the set of positive integers. 
The following theorem holds

THEOREM 2. If the function $x:\left[t_{0}, \infty\right) \rightarrow \mathbf{R}^{n}$ is

(i) uniformly continuous and bounded on $\left[t_{0}, \infty\right)$,

(ii) $T$-stable (according to Definition 2), then $x(t)$ is asymptotically almost periodic.

The concept of asymptotic almost periodicity was introduced by Fréchet [3], who also gave the equivalent condition: the continuous function $x$ : $\left[t_{0}, \infty\right) \rightarrow \mathbf{R}^{n}$ is asymptotically almost periodic if and only if for every sequence $\left\{\alpha_{n}\right\}$, with $\alpha_{n} \rightarrow \infty$ for $n \rightarrow \infty$, there is a subsequence $\left\{\alpha_{n}^{\prime}\right\}$ such that $\left\{x\left(t+\alpha_{n}^{\prime}\right)\right\}$ is uniformly convergent on $\left[t_{0}, \infty\right)$. Using this condition, the proof of Theorem 2 is similar to that of Theorem 1 and is therefore omitted.

3. Periodic systems: comparison between Liapunov stability and $T$-stability. Let us consider a periodic system of $n$ differential equations

$$
\dot{x}=f(t, x)
$$

where $x, f \in \mathbf{R}^{n}$ and the function $f(t, x)$ is defined and continuous on $\mathbf{R} \times \Omega$, where $\Omega$ is a connected open set of $\mathbf{R}^{n}$, and $f(t+T, x) \equiv f(t, x)$, for some period $T>0$. Moreover, let the right-hand side of (3.1) be smooth enough to ensure the uniqueness of the solutions. Then the maximal solution of (3.1) through $\left(t_{0}, x_{0}\right) \in \mathbf{R} \times \Omega$ will be denoted by $\varphi\left(t, t_{0}, x_{0}\right)$ and by definition it is $\varphi\left(t_{0}, t_{0}, x_{0}\right)=x_{0}$.

An equivalent condition of $T$-stability for a solution $x(t)$ of (3.1) defined for $t \geqslant t_{0}$, is given by the following

LEMMA. A necessary and sufficient condition for a solution $x:\left[t_{0}, \infty\right) \rightarrow \mathbf{R}^{n}$ of the T-periodic system (3.1) to be T-stable is

$$
\begin{gathered}
(\forall \varepsilon>0)(\exists \delta>0)\left(\forall m \in \mathbf{N}:\left\|x\left(t_{0}\right)-x\left(t_{0}+m T\right)\right\|<\delta\right)\left(\forall t \geqslant t_{0}\right) \\
\|x(t)-x(t+m T)\|<\varepsilon .
\end{gathered}
$$

Condition (3.2) is obtained from (2.2), putting $n=0$. Let us show that condition (3.2) implies $T$-stability with the same pair $(\varepsilon, \delta(\varepsilon))$. Let $\bar{n}, \bar{m} \in \mathbf{N}$ $(\bar{n} \geqslant \bar{m})$ be given such that $\left\|x\left(t_{0}+\bar{n} T\right)-x\left(t_{0}+\bar{m} T\right)\right\|<\delta$. With the change of variable: $t \rightarrow t+\bar{m} T$ and for the particular value $m=\bar{n}-\bar{m} \geqslant 0$, we obtain from (3.2) the following relation: $\left\|x\left(t_{0}+\bar{m} T\right)-x\left(t_{0}+\bar{n} T\right)\right\|<\delta$ $\Rightarrow \forall t \geqslant t_{0},\|x(t+\bar{m} T)-x(t+\bar{n} T)\|<\varepsilon$, which proves the lemma.

Let us suppose that the system (3.1) possesses a solution $x(t)$ bounded in the future, i.e. defined for $t \geqslant t_{0}$ and such that $x(t) \in K \subset \Omega$ for $t \geqslant t_{0}$, where $K$ is a compact set of $\mathbf{R}^{n}$. Then $x(t)$ is uniformly continuous on $\left[t_{0}, \infty\right)$, due to the continuity and periodicity of the right-hand side of (3.1), and therefore taking into account the lemma, Theorem 2 of $\$ 2$ can be reformulated in the following way

THEOREM 3. Let $x(t)$ be a solution of the T-periodic system (3.1) such that 
(i) $x(t)$ is bounded in the future,

(ii)

$$
\begin{gathered}
(\forall \varepsilon>0)(\exists \delta>0)\left(\forall m \in \mathrm{N}:\left\|x\left(t_{0}\right)-x\left(t_{0}+m T\right)\right\|<\delta\right)\left(\forall t \geqslant t_{0}\right) \\
\|x(t)-x(t+m T)\|<\varepsilon .
\end{gathered}
$$

Then $x(t)$ is asymptotically almost periodic.

Let us now compare $T$-stability with Liapunov stability, which is defined as follows: the solution $x:\left[t_{0}, \infty\right) \rightarrow \mathbf{R}^{n}$ is Liapunov stable if, for every $\varepsilon>0$ and every $t_{0}^{\prime} \geqslant t_{0}$ there is a $\delta=\delta\left(\varepsilon, t_{0}^{\prime}\right)>0$ such that for any $x_{0}^{\prime}$ with $\| x_{0}^{\prime}-$ $x\left(t_{0}^{\prime}\right) \|<\delta$ there follows $\left\|x(t)-\varphi\left(t, t_{0}^{\prime}, x_{0}^{\prime}\right)\right\|<\varepsilon$ for all $t \geqslant t_{0}^{\prime}$. Then taking $t_{0}^{\prime}=t_{0}$ and $x_{0}^{\prime}=x\left(t_{0}+m T\right), m=1,2, \ldots$, we obtain condition (3.2), which is equivalent to $T$-stability (remark that $\delta=\delta\left(\varepsilon, t_{0}\right)$ depends only on $\varepsilon$, because $t_{0}$ has to be considered as fixed). This shows that for periodic systems $T$-stability is weaker than Liapunov stability. In fact, $T$-stability is a conditional stability, i.e. the solution $x(t)$ is stable at $t_{0}$ only with respect to the particular solutions corresponding to the initial conditions $\left(t_{0}, x\left(t_{0}+m T\right)\right)$, i.e. the solutions $x(t+m T), m=1,2, \ldots$ Therefore the following corollary of Theorem 3 holds

COROllary. If a solution $x(t)$ of the periodic system (3.1) is bounded in the future and Liapunov stable, then $x(t)$ is asymptotically almost periodic.

This result shows that the condition of uniform stability in the sense of Liapunov (which is always assumed in the literature to assure the asymptotic almost periodicity of a solution bounded in the future, beginning with $\mathrm{A}$. Halanay [4, p. 486]) is unnecessarily strong. In fact, for a nontrivial solution of a periodic system, stability and uniform stability are not equivalent (see [5, p. 42]).

REMARK. The results obtained in the present section can be extended to periodic systems of differential equations for which the uniqueness of solutions does not hold.

\section{REFERENCES}

1. A. Markov, Stabilität im Liapounoffschen Sinne und Fastperiodizität, Math. Z. 36 (1933), 708-738.

2. A. M. Fink, Almost periodic differential equations, Lecture Notes in Math., vol. 377, Springer-Verlag, New York, 1974.

3. M. Fréchet, Les fonctions asymptotiquement presque-périodiques, Rev. Sci. 79 (1941), 341-354.

4. A. Halanay, Differential equations: Stability, oscillations, time lags, Academic Press, New York, 1966.

5. T. Yoshizawa, Stability theory and the existence of periodic solutions and almost periodic solutions, Appl. Math. Sci. 14, Springer-Verlag, New York, 1975.

Istituto di Matematica, Università degli Studi, 43100 Parma, Italy 